\title{
On the relationship between bond correction factors and elemental mean excitation energies
}

Sauer, Stephan P. A.; Sabin, John R.; Oddershede, Jens

Published in:

Nuclear Instruments and Methods in Physics Research Section B: Beam Interactions with Materials and Atoms

DOI:

10.1016/j.nimb.2020.04.021

Publication date:

2020

Document version

Early version, also known as pre-print

Document license:

Unspecified

Citation for published version (APA):

Sauer, S. P. A., Sabin, J. R., \& Oddershede, J. (2020). On the relationship between bond correction factors and elemental mean excitation energies. Nuclear Instruments and Methods in Physics Research Section B: Beam Interactions with Materials and Atoms, 474, 6-9. https://doi.org/10.1016/j.nimb.2020.04.021 


\title{
On the relationship between bond correction factors and elemental mean excitation energies
}

\author{
Stephan P.A. Sauer ${ }^{1}$, John R. Sabin ${ }^{3,2}$ and Jens Oddershede ${ }^{2,3^{*}}$ \\ 1. Department of Chemistry, University of Copenhagen, 2100 Copenhagen, Denmark \\ 2. Department of Physics, Chemistry and Pharmacy, University of Southern Denmark, 5230 Odense M, \\ Denmark \\ 3. Departments of Physics and Chemistry, University of Florida, Gainesville, Florida 32611, USA.
}

*Corresponding author, email: jod@sdu.dk.

\begin{abstract}
We have investigated the relationship between two methods to obtain mean excitation energies of large samples using a modified Bragg rule, one method using Bragg's rule to determine elemental mean excitation energies from experimental stopping power data, and another method going the opposite way, that is determining compound mean excitation energies from theoretical elemental mean excitation energies and bond correction factors. We show how to obtain bond correction factors from elemental mean excitation energies and vice versa. The comparison leads to insight into the importance of the effect of chemical binding on the experimentally determined elemental mean excitation energies. We also introduce the concept of atomic correction factor that links theoretical, gas phase atomic mean excitation energies to elemental mean excitation energies.
\end{abstract}

Keywords

Mean excitation energies, Bragg's rule, particle therapy, stopping power

\section{Introduction}

The mean excitation energy, I, is the most central materials constant determining the slowing down of fast, high energy particles in matter $[1,2]$. As matter often are complicated substances in terms of molecular structure and compositions of those [3,4], the determination of the mean excitation energies will have to rely upon a method that can calculate I of a sample from the parts making up the sample. Here, Bragg's additivity rule [5] is the most suitable tool and extensive use of it has been made over the years $[6,7]$.

However, in its original form Bragg's rule has some shortcomings as it disregards the chemical binding and the phase differences of the parts making up the large sample and the sample itself. Recently, two different approaches have been suggested that might overcome these difficulties by modifying Bragg's original rule, the introduction of elemental mean excitation energies [8] and the concept of bond correction factors [9]. The purpose of this note is to compare the two methods and to show how they are related.

\section{Elemental mean excitation energies}

Recently, Bär et al. [8] have introduced the concept of elemental mean excitation energies $I_{\text {elem, } j}$ as a tool to calculate mean excitation energies of large media, $I_{\text {med }, i}$, for which you know the elemental composition, such as human tissues [3,4]. To this end they are using Bragg's rule [5] expressed as 


$$
\ln I_{m e d, i}=\sum_{j=1}^{N} \lambda_{m e d, i j} \operatorname{lnI} I_{e l e m, j}
$$

Here, $\mathrm{N}$ is the number of elements and $\lambda_{m e d, i j}$ is the fraction of electrons from the $\mathrm{j}$-th element in the $\mathrm{i}$-th medium given by

$$
\lambda_{\text {med }, i j}=\frac{w_{\text {med }, i j}\left({ }^{Z_{j}} / A_{j}\right)}{(Z / A)_{m e d, i}}
$$

where $w_{\text {med, } i j}$ is the elemental weight of the $\mathrm{j}$-th element in the $\mathrm{i}$-th medium and $Z_{j}$ and $A_{j}$ are the atomic number and molar mass, respectively, of the $j$-th element. Correspondingly, $(Z / A)_{m e d, i}$ is number of electrons per unit mass in the $\mathrm{i}$-th medium (in $\mathrm{mol} / \mathrm{g}$ ).

Bär et al. [8] is making use of Eq. (1) in two ways. First, to calculate the elemental mean excitation energies from mean excitation energies of a range of media using both experimental and theoretical values [10-15] for $I_{\text {med, } i}$, that is, inverting Eq. (1). They do so, using statistical mathematical methods. Both the elemental mean excitation energies and their uncertainties are reported. Two optimized sets of elemental mean excitation energies are obtained, one for gases and one for liquids and solids. By constraining the fit to obey Eq. (1) they are including effects of chemical bonding and phase in the elemental mean excitation energies. Thus, the atomic elemental mean excitation energies are no longer identical to pure atomic mean excitation energies as can be seen in Table 1.

Table 1. Comparison of gas phase elemental $[8,10]$ and theoretical [16] atomic mean excitation energies (in $\mathrm{eV})$.

\begin{tabular}{|l|l|l|l|}
\hline Element & $\begin{array}{l}\text { Berger and Seltzer } \\
{[10]^{\mathrm{a}}}\end{array}$ & Bär et al. [8] ${ }^{\mathrm{a}, \mathrm{b}}$ & Sauer et al. [16 $]^{\mathrm{c}}$ \\
\hline $\mathrm{H}$ & 19.20 & $21.54(0.74)$ & 14.99 \\
\hline $\mathrm{C}$ & 70.00 & $66.75(1.08)$ & 65.94 \\
\hline $\mathrm{N}$ & 82.00 & $79.59(1.15)$ & 81.64 \\
\hline $\mathrm{O}$ & 97.00 & $95.17(1.01)$ & 97.93 \\
\hline
\end{tabular}

${ }^{\mathrm{a}}$ Elemental mean excitation energies obtained from data for a range of media.

${ }^{\mathrm{b}}$ The number in parenthesis is the uncertainty in eV computed by Bär et al. [8].

${ }^{\mathrm{C} T h e o r e t i c a l ~ R a n d o m-P h a s e ~ c a l c u l a t i o n ~ f o r ~ a t o m s . ~}$

Table 1 shows that the biggest difference between the atomic and elemental mean excitation energies for atoms from Bär et al. [8] is found for $\mathrm{H}$. This is not so surprising since we have shown [17], that the smallest error in the use of Bragg's rule is obtained when the hydrogen fragments are $\mathrm{H}_{2}$ and not $\mathrm{H}$. In fact, the mean excitation energy for $\mathrm{H}_{2}$ is $19.47 \mathrm{eV}$ [17], which is much closer to the elemental mean energies for $\mathrm{H}$ in Table 1. The other atoms also show differences between the atomic and elemental mean excitation energies. Only for $\mathrm{C}$ does the theoretical, gas phase value for I lie inside the error bars quoted by Bär et al. [8]. The earlier estimates of elemental mean excitation energies by Berger and Seltzer [10] for $\mathrm{N}$ and $\mathrm{O}$ are more like the theoretical atomic mean excitation energies. 
The second application of Eq. (1) by Bär et al. [8] was to calculate mean excitation energies for 70 human tissues using the relative atomic weights of the tissues $[3,4]$, thus demonstrated the usefulness of the method to produce mean excitation energies that may be used in radiative treatment of patients.

\section{Bond correction factors}

The use of bond correction factors for the calculation of molecular mean excitation energies also make use of Bragg's rule [5] for mean excitation energies. It adds a new term to the atomic Bragg rule to account for chemical binding so that it becomes [9]

$$
N_{e} \ln I=\sum_{i=1}^{N_{a}} N_{e, i} \ln I_{i}+\sum_{k=1}^{N_{b}} N_{e, k} \ln f_{k}
$$

where $N_{e}$ is the number of electrons in the molecule, $N_{e, k}$ is the number of electrons in the k-th bond and $N_{e, i}$ is the number of electrons in the i-th atom, thus

$$
\sum_{i=1}^{N_{a}} N_{e, i}=N_{e}
$$

Here, $N_{a}$ and $N_{b}$ are the number of atoms and bond in the molecule, respectively. The dimensionless bond correction factor for the k-th bond, $f_{k}$, is introduced to correct for the lack of chemical bonding if only the first term was included in Eq. (3).

Eq. (3) may be reformulated to [9]

$$
N_{e} \ln I=\sum_{i}^{N_{a}} N_{e, i, n o n-b o n d i n g} \ln I_{i}+\sum_{i, j=1}^{N_{a}} n_{i \leftrightarrow j} \ln \left(I_{i} f_{i \leftrightarrow j}\right)
$$

where the $i \leftrightarrow j$ symbol indicates "a bond between atom $\mathrm{i}$ and atom $\mathrm{j}$ ", and $n_{i \leftrightarrow j}$ is a degeneracy factor equal to 1 for a single bond, 2 for a double bond etc. The first sum only extends over the electrons that do not take part in the bond. From this equation we see that we may interpret the bond correction factor as a dimensionless quantity that multiplies the mean excitation energies of the atoms that take part in the bond, thereby accounting for the effect of chemical binding.

In the original application of bond correction factors [9] we used Eq. (3) to compute bond correction factors from known atomic and molecular mean excitation energies. We obtained a universal set of bond correction factors that could be applied to the calculation of molecular mean excitation energies, incorporating the effect of chemical bonding, for large molecules for which a direct calculation of the mean excitation energy is not possible.

However, we could also have used Eqs. (3) and (4) more along the lines outlined by Bär et al. [8]. If I in Eq. (3) had been an experimental mean excitation energy, then the bond correction factors that were determined from Eq. (3) would include all the effects that were included in the experimental excitation energies such as chemical bonding and phase effects. As an example, let us consider liquid $\mathrm{H}_{2} \mathrm{O}$, for which the ICRU $90[18,19]$ recommended value for I is $78 \pm 2 \mathrm{eV}$. Inserting this value for I and the atomic mean excitation from Table 1 in Eq. (4) we find that $f_{O \leftrightarrow H}=1.47 \pm 0.07$. Only including chemical bonding in a single, gas phase water molecule, we [9] found that $f_{O \leftrightarrow H}=1.22$. Thus, including phase effect increases the bond correction factor with ca. $21 \%$. As $f_{O \leftrightarrow H}=1$ when there is no bonding, we see that the effects of phase and chemical bonding are of the same order of magnitude for $\mathrm{H}_{2} \mathrm{O}$. 


\section{Calculation of bond correction factors from elemental mean excitation energies}

The water example illustrates how one may calculate bond correction factors that include effects of both bonding and phase from the elemental mean excitation energies. For any molecule, we find from Eqs. (1) and (4) that

$\sum_{i=1}^{N_{a}} N_{e, i} \ln I_{\text {elem }, i}=\sum_{i=1}^{N_{a}} N_{e, i, n o n-b o n d i n g} \ln I_{i}+\sum_{i, j=1}^{N_{a}} n_{i \leftrightarrow j} \ln \left(I_{i} f_{i \leftrightarrow j}^{e x p}\right)$

where $f_{i \leftrightarrow j}^{\exp }$ then becomes the bond correction factor that will reproduce the experimental mean excitation energy that was inserted as the left-hand sides of both Eqs. (1) and (4) in order to obtain Eq. (6).

Proceeding serially, we may now calculate $f_{i \leftrightarrow j}^{\exp }$ using Eq. (6). We start out with molecules with only one unknown bond, inserting on the left-hand side of this equation the elemental mean excitation energies from Bär et al. [8]. Next, we then calculate "experimental" bond correction factors for molecules with more bond but with only one unknown bond correction factors. A few examples of the application of this method are illustrated in Table 2, starting out with molecules with only one unknown bond correction factor. We are showing bond correction factor including all corrections to the atomic Bragg's rule, $f_{i \leftrightarrow j}^{\text {exp }}$, as well as the factors that were obtained including only chemical bonding, $f_{i \leftrightarrow j}^{b o n d}[9]$.

Table 2. Comparison of bond correction factors per electron obtained from liquid-solid phase elemental mean excitation energies, $f_{X \leftrightarrow Y}^{\text {exp }}$, and bond correction factors for single, gas phase molecules, $f_{X \leftrightarrow Y}^{b o n d}$ [9].

\begin{tabular}{|l|l|l|l|l|l|}
\hline Bond, $\mathrm{X} \leftrightarrow \mathrm{Y}$ & Molecule & $\mathrm{I}^{\mathrm{exp}}(\mathrm{eV})^{\mathrm{a}}$ & $f_{X \leftrightarrow Y}^{\text {exp }} \mathrm{b}$ & $f_{X \leftrightarrow Y}^{\text {bondc }}$ & $\Delta \mathrm{f}(\%)^{\mathrm{d}}$ \\
\hline $\mathrm{O}-\mathrm{H}$ & $\mathrm{H}_{2} \mathrm{O}$ & 78.73 & 1.48 & 1.22 & 21 \\
\hline $\mathrm{C}-\mathrm{H}$ & $\mathrm{CH}_{4}$ & 48.30 & 1.19 & 1.25 & -5 \\
\hline $\mathrm{N}-\mathrm{H}$ & $\mathrm{NH}_{3}$ & 53.82 & 1.17 & 1.20 & -3 \\
\hline $\mathrm{C}=\mathrm{O}$ & $\mathrm{CO}_{2}$ & 99.11 & 1.18 & 1.07 & 10 \\
\hline $\mathrm{N}-\mathrm{O}$ & $\mathrm{N}_{2} \mathrm{O}$ & 87.57 & 1.01 & 0.96 & 5 \\
\hline $\mathrm{F}-\mathrm{H}$ & $\mathrm{HF}$ & 113.89 & 2.49 & 1.26 & 98 \\
\hline $\mathrm{C}-\mathrm{C}$ & $\mathrm{C}_{2} \mathrm{H}_{6}$ & 52.53 & 1.25 & 1.12 & 12 \\
\hline $\mathrm{C}=\mathrm{C}$ & $\mathrm{C}_{2} \mathrm{H}_{4}$ & 58.34 & 1.17 & 1.07 & 9 \\
\hline $\mathrm{C} \equiv \mathrm{C}$ & $\mathrm{C}_{2} \mathrm{H}_{2}$ & 66.76 & 1.23 & 1.05 & 17 \\
\hline $\mathrm{C}-\mathrm{N}$ & $\mathrm{CH}_{3} \mathrm{NH}$ & 55.78 & 1.85 & 1.09 & 71 \\
\hline $\mathrm{C}=\mathrm{N}$ & $\mathrm{CH}_{2} \mathrm{NH}$ & 62.42 & 1.30 & 1.07 & 21 \\
\hline $\mathrm{C}-\mathrm{O}$ & $\mathrm{CH}_{3} \mathrm{OH}$ & 68.91 & 1.54 & 1.08 & 43 \\
\hline $\mathrm{C}-\mathrm{F}$ & $\mathrm{CH}_{3} \mathrm{~F}$ & 84.60 & 1.58 & 1.14 & 39 \\
\hline
\end{tabular}

${ }^{\mathrm{a}}$ Calculated from Eq. (1) using the elemental bond mean excitation energies of Table 3 of Bär et al. [8]. Please note that the elemental mean excitation energy of $\mathrm{H}$ is $22.70 \mathrm{eV}$ and not $22.07 \mathrm{eV}$ as listed in Table 3 of ref. [8] (E. Bär and H. Bouchard, private communication).

${ }^{b}$ Calculated from Eq. (6) using liquid-solid phase elemental mean excitation energies [8] and gas phase atomic mean excitation energies $\mathrm{I}_{\mathrm{i}}$ from ref. [16].

${ }^{\mathrm{c}}$ From ref. [9].

${ }^{\mathrm{d}} f_{X \leftrightarrow Y}^{\exp }-f_{X \leftrightarrow Y}^{b o n d s}$ in per cent. 
Table 2 shows - not unexpectedly - that for nearly all molecules the bond corrections factors that include all corrections to the atomic Bragg rule are larger than the factors including only the effect of chemical binding. However, the differences between the two sets of bond correction factors vary considerably, and it is not possible from the small sample of molecules in Table 2 to deduct simple rules for predicting the size of the bond correction factors that will be needed to calculate "experimental mean excitation energies" from Eq. (3). On the other hand, the method outlined in Table 2 can in principle be applied to any molecule that can be constructed from the elemental liquid and solid elemental mean excitation energies given in Table 3 of Bär et al. [8]. We thus have a method to calculate of $f_{X \leftrightarrow Y}^{\exp }$ for any bond between atoms for which Bär et al. [8] have reported elemental mean excitation energies.

\section{Atomic correction factors}

However, in order to apply Eq. (3) to calculate the experimental mean excitation energy for any molecule we must know the bonding pattern of the molecule. This is not the case for human tissues that generally have rather complicated - and often unknown - molecular structure. For tissues only the elemental composition is known [3,4] which also was the motivation for Bär et al. [8] to determine the elemental mean excitation energy for each atomic substance so that they could use Eq. (1) to obtain the mean excitation energy for any tissue for which you know the elemental composition.

Translated into a concept of factors multiplying gaseous mean excitation energies we would need to have atomic correction factors, $F_{i}$ rather than bond correction factors if we were to calculate "experimental mean excitation energies" for compounds for which we only know the elemental composition. A trivial way to produce such factors would be to divide the elemental mean excitation energies from Bär et al. [8] by the atomic mean excitation energies [16]

$I_{\text {elem }, i}=F_{i} I_{\text {atom }, i}$

The $F_{i}$ factors derived from the elemental mean excitation energies from Bär et al. [8] are listed in Table 3.

Table 3. Atomic correction factors for elements for which Bär et al. [8] have reported elemental mean excitation energies in the liquid and solid phase.

\begin{tabular}{|l|l|l|l|}
\hline Atom i & $I_{\text {elem }, i}[8]$ & \multicolumn{1}{|c|}{$I_{\text {atom }, i}[16]$} & $F_{i}^{\mathrm{a}}$ \\
\hline $\mathrm{H}$ & 22.70 & 14.99 & 1.51 \\
\hline $\mathrm{C}$ & 79.91 & 65.94 & 1.21 \\
\hline $\mathrm{N}$ & 77.91 & 81.64 & 0.95 \\
\hline $\mathrm{O}$ & 107.44 & 97.93 & 1.10 \\
\hline $\mathrm{F}$ & 136.24 & 116.52 & 1.17 \\
\hline $\mathrm{Al}$ & 191.69 & 132.19 & 1.45 \\
\hline $\mathrm{Si}$ & 150.47 & 140.82 & 1.07 \\
\hline $\mathrm{P}$ & 199.39 & 151.61 & 1.32 \\
\hline $\mathrm{Cl}$ & 175.13 & 174.92 & 1.00 \\
\hline
\end{tabular}

${ }^{\mathrm{a} C a l c u l a t e d}$ from Eq. (7) 
As the experimentally derived mean excitation energies generally are larger than the atomic mean excitation energies, the atomic correction factors are nearly always larger than 1 - but not larger than 1.5. However, as was the case for the bond correction factors that were used to reproduce the experimental mean excitation energies it is difficult to see a pattern in the variation in the size of the atomic correction factors.

\section{Calculation of elemental mean excitation energies from bond correction factors}

We have so far showed how to calculate atom and bond correction factors from elemental mean excitation energies, that is correction factors to the atomic Bragg rule needed to obtain experimental mean excitation energies. However, one would like to be able to go the other way, i.e. to compute elemental mean excitation energies from bond correction factors. This can be done by inserted Eq. (7) into Eq. (6) whereby we find that

$\sum_{i=1}^{N_{a}} N_{e, i} \ln F_{i}^{X}=\sum_{i, j=1}^{N_{a}} n_{i \leftrightarrow j} \ln \left(f_{i \leftrightarrow j}^{X}\right)$

where the superscripts on $F_{i}$ and $f_{i \leftrightarrow j}$ in Eq. (8) could be exp in which case we will have an equation for the experimental atom and bond correction factors, that is Eq. (6). However, it could also be bond in which case Eq. (8) shows how to calculate atomic correction factors including only chemical bonding from $f_{i \leftrightarrow j}^{b o n d}$. Hence, Eq. (8) makes it possible to calculate atomic correction factors, and thus from Eq. (7) elemental mean excitation energies, from bond correction factors.

As any molecule has more atoms than bonds, we need more molecules consisting of the same atoms to make use of Eq. (8) to calculate $F_{i}^{b o n d}$ from $f_{i \leftrightarrow j}^{b o n d}$. To illustrate this process, let us consider linear hydrocarbons for which we have two kinds of bonds, $\mathrm{C}-\mathrm{H}$ and $\mathrm{C}-\mathrm{C}$ in alkanes, $\mathrm{C}-\mathrm{H}$ and $\mathrm{C}=\mathrm{C}$ in alkenes and $\mathrm{C}$ $\mathrm{H}$ and $\mathrm{C} \equiv \mathrm{C}$ in alkynes. We thus need two equations to determine the two atomic correction factors for hydrogen and carbon for the hydrocarbons. It turns out that it does not matter which two hydrocarbons we pick for either of the three sets of hydrocarbons. We can thus obtain an analytic expression for the atomic correction factor which is independent of the number of carbons in the chain. The results are summarized in Table 4.

Table 4. Atomic correction factors for hydrocarbons calculated from Eq. (8) using the bond correction factors $f_{X-Y}$ from ref. [9].

\begin{tabular}{|l|c|l|c|l|}
\hline Molecule & $F_{H}^{\text {bond }}$, analytical & $F_{H}^{\text {bond }}$, numerical & $F_{C}^{\text {bond }}$, analytical & $F_{C}^{\text {bond }}$, numerical \\
\hline Alkanes, $\mathrm{C}_{\mathrm{n}} \mathrm{H}_{2 \mathrm{n}+2}$ & $f_{C-H}^{2} / f_{C-C}$ & 1.40 & $\left(f_{C-C}\right)^{2 / 3}$ & 1.08 \\
\hline Alkenes, $\mathrm{C}_{\mathrm{n}} \mathrm{H}_{\mathrm{n}+2}$ & $f_{C-H}^{2} / f_{C-C}$ & 1.40 & $\left(f_{C=C} f_{C-C}^{2}\right)^{1 / 6}$ & 1.05 \\
\hline Alkynes, $\mathrm{C}_{\mathrm{n}} \mathrm{H}_{2}$ & $f_{C-H}^{2} / f_{C-C}$ & 1.40 & $\left(f_{C \equiv C} f_{C-C}\right)^{1 / 6}$ & 1.07 \\
\hline
\end{tabular}

As the bond correction factors used to calculate the atomic equivalents only include the effect of chemical bonding [9], the same will be the case for the computed $F_{i}^{b o n d}$. Comparing the atomic corrections factors including all corrections in Table 3 with the ones in Table 4 we see that the effect of chemical bonding gives the leading contribution to the hydrogen correction factor but not to $F_{C}$. 
The results in Table 4 also show that the hydrogen correction factor is the same for all three series of hydrocarbon but that this is not the case for the correction factor for carbon. The background for this difference is that an "atom-in-a-molecule" is not a transferable quantity in the same way as a molecular bond is. The hydrogen atoms are in a similar binding situation in the three sets of hydrocarbons but this not the case for carbons. Hence, we find different values for the three carbon correction factors.

The elemental atomic mean excitation energies determined by Bär et al. [8] are designed to be used for a range of compounds for which the atoms are in many different binding situations. The error bars on the reported [8] elemental mean excitation energies should hopefully incorporate this uncertainty. The reason for $F_{c}^{\text {bond }}$ in Table 4 being rather different from the one included all corrections in Table 3 is probably that the sampling used to determine the carbon elemental mean excitation energies in liquids and solids by Bär et al. [8] include many bonding situations for $C$ that are rather different than the ones one finds in hydrocarbons.

\section{Summary}

We have compared two different ways of calculating mean excitation energies for large molecules and samples using a modified version of Bragg's rule [5], elemental mean excitation energies [8] and bond and atom correction factors [9]. We have reported how to obtain bond and atom correction factors from elemental mean excitation energies, that is bond and atom correction factors that include all the effect that are included in the experimentally derived elemental mean excitation energies. For the few molecules for which we have calculated bond correction factors included both chemical bonding and all the effects included by Bär et al. [8] we find rather large differences in the relative importance of the effects of chemical bonding. This difference is probably caused by the sampling procedure used by Bär et al. [8] to determine the recommended set of elemental mean excitation energies.

We have also derived an equation that allows determination of elemental mean excitation energies from bond correction factors and we have demonstrated how this works for the example of linear hydrocarbons. This approach makes it possible to determine how much molecular binding contributes to the experimentally derived elemental mean excitation energies.

We conclude that it is relatively simple to relate to two approaches to each other and that the methods contribute with complementary knowledge on the determination of mean excitation energies for large samples and molecules.

\section{References}

[1] H. Bethe, Zur Theorie des Durchgangs Schneller Korpuskularstrahlen durch Materie, Ann. Phys. 397 (1930) 325-400.

[2] M. Inokuti, Inelastic collisions of fast charged particles with atoms and molecules - The Bethe theory revisited, Rev. Mod. Phys. 43 (1971) 297-347.

[3] H.Q. Woodard, D.R. White, The composition of body tissues. Br. J. Radiol. 59 (1986) 1209-1218.

[4] D.R. White, H.Q. Woodard, S.M. Hammond, Average soft-tissue and bone models for use in radiation dosimetry, Br. J. Radiol. 60 (1987) 907-913. 
[5] W. H. Bragg, R. Kleeman, On the alpha particle of radium and their loss of range in passing through various atoms and molecules, Philos. Mag. 10 (1905) 318-340.

[6] D.I. Thwaites, Departures from Bragg's rule of stopping power additivity for ions in dosimetric and related materials, Nucl. Instr. Meth. Phys. Res. Sect. B 69 (1992) 53-63.

[7] L.E. Porter, Additivity studies of the stopping powers of eleven halogenated hydrocarbons for protons and $\alpha$ particles, Phys. Rev. A (1994) 50 2397-2404.

[8] E. Bär, P. Andreo, A. Lalonde, G. Royle, H. Bouchard, Optimized I-values for use with the Bragg additivity rule and their impact on proton stopping power and range uncertainty, Phys. Med. Biol. 63 (2018) 165007.

[9] S.P.A. Sauer, J.R. Sabin, J. Oddershede, Bond correction factors and their applications to the calculation of molecular mean excitation energies, Nucl. Instr. Meth. Phys. Res. B 468 (2020) 28-36.

[10] M.J. Berger, S.M. Seltzer, Stopping Powers and Ranges of Electrons and Positrons, US Govt. Printing Office, Washington D.C. NBS IR 82-2550A (1982).

[11] H. Bichsel, T. Hiraoka, Energy loss of $70 \mathrm{MeV}$ protons in elements, Nucl. Instr. Meth. Phys Res. B 66 (1992) 345-351.

[12] T. Hiraoka, K. Kawashima, K. Hoshino, A. Fukumu, H. Bichsel, Energy loss of 70 MeV protons in organic polymers, Med. Phys. 20 (1993) 135-141.

[13] Hiraoka, K. Kawashima, K. Hoshino, H. Bichsel, Energy loss of $70 \mathrm{MeV}$ protons in tissue-substitute materials, Phys. Med. Biol. 39 (1994) 983-991.

[14] H. Bichsel, T. Hiraoka, K. Omata, Aspects of fast-ion dosimetry, Radiat. Res. 153 (2000) 208-219.

[15] Y. Kumazaki, T. Akagi, T. Yanou, T. Suga, Y. Hishikawa, T. Teshima, Determination of the mean excitation energy of water from proton beam range, Radiat. Meas. 42 (2007) 1683-1691.

[16] S.P.A. Sauer, J.R. Sabin, J. Oddershede, Z-dependence of mean excitation energies for second and third row atoms and their ions, J. Chem. Phys. 148 (2018) 174307.

[17] S.P.A. Sauer, J.R. Sabin, J. Oddershede, Test of the validity of Bragg's rule for mean excitation energies of small molecules and ions, Nucl. Instr. Meth. Phys. Res. B 444 (2019) 112-116.

[18] ICRU Report, Key data for ionizing radiation dosimetry: Measurement standards and applications (Bethesda, MD: International Commission on Radiation Units and Measurements) 90 (2014).

[19] P. Andreo, J. Wulff, D. Burns, H. Palmans, Consistency in reference radiotherapy dosimetry: Resolution of an apparent conundrum when $60 \mathrm{co}$ is the reference quality for charged-particle and photon beams, Phys. Med. Biol. 58 (2013) 6593. 
